\title{
Technique for "open sternal" chest closure in patients with assist devices and transplant recipients
}

\author{
Velu Balasubramanian, MD, ${ }^{\mathrm{a}}$ and Jay K. Bhama, $\mathrm{MD}^{\mathrm{b}, \mathrm{c}}$
}

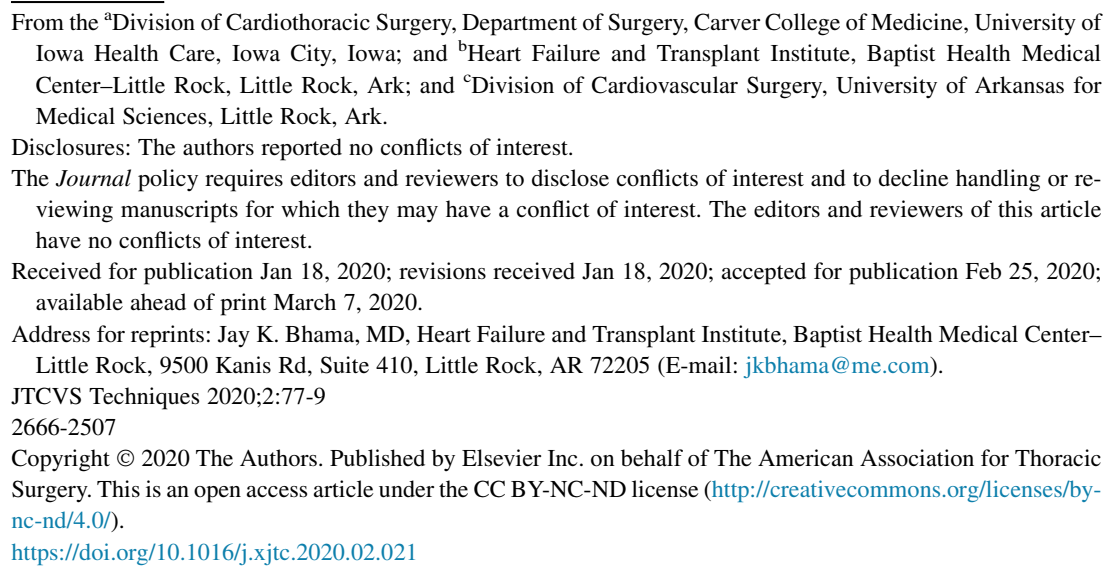

$\checkmark$ Video clip is available online.

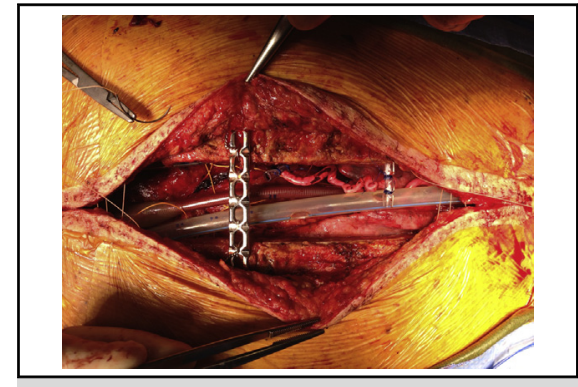

Open sternal chest closure technique.

CENTRAL MESSAGE

A novel technique is described to maintain secure sternal separation and a biological barrier after cardiac transplant or LVAD implantation.

See Commentaries on pages $\mathbf{8 0}$ and $\mathbf{8 2 .}$
Selection criteria in both cardiac transplantation and left ventricular assist device (LVAD) placement are expanding, leaving patients at risk for problems that require the sternum to be left open after surgery, such as coagulopathy or right ventricular dysfunction. Standard approaches for keeping the sternum open rely on plastic syringe struts to spread the sternum and a nonbiological barrier (ie, esmarch). A technique is described to achieve sternal separation while maintaining a biological barrier, which has been used selectively for delayed sternal closure (Video 1). Although the technique may be used for any patient after cardiac surgery, we have predominately used it in transplant recipients and patients with LVADs.

\section{SURGICAL TECHNIQUE}

The technique involves wound approximation over the unapproximated sternum. If there is cardiac compression, this is coupled with limited skin flaps and sternal bridging using an orthopedic plate (Synthes GMBH, Oberdorf, Switzerland) secured to the sternum with wire (Figure 1). The wire is passed through the sternum on either side and then through the desired hole in the plate to create the separation required before securing. This is only required in patients with significant hemodynamic deterioration when the sternal retractor is removed. If the right ventricle contacts the sternum, we place a piece of Gelfoam (Pharmacia and Upjohn Co, Kalamazoo, Mich) to serve as a barrier between the heart and the sternal edge. We avoid gauze over the heart because it can exert pressure on the right ventricle. Wound approximation is assisted by interrupted vertical

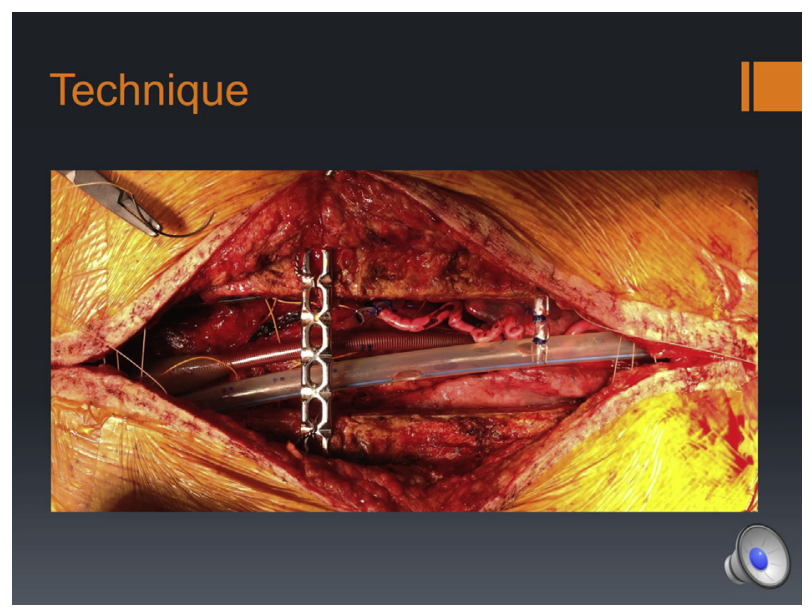

VIDEO 1. Video describing the open sternal chest closure technique. Video available at: https://www.jtcvs.org/article/S2666-2507(20)30127-9/ fulltext. 


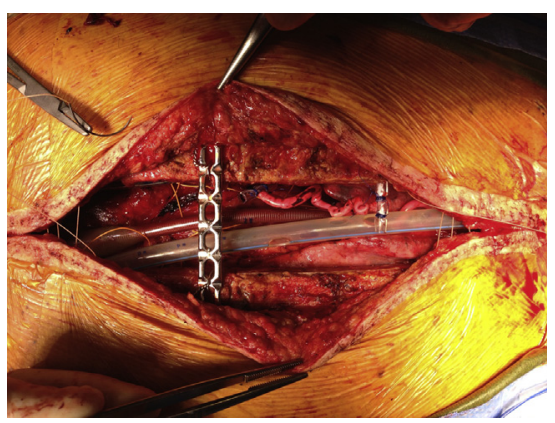

FIGURE 1. Intraoperative photograph demonstrating the "open sternal" chest closure technique including sternal stabilization with an orthopedic plate and subcutaneous tissue closure over the open sternum.

mattress stitches sequentially tied approximating the edges allowing for subdermal closure with absorbable suture followed by skin staples and removal of the mattress sutures. The wound is then covered directly with an incise drape.

\section{CLINICAL SUMMARY}

This technique was applied in 29 patients from February 2009 to December 2017 (Table 1), representing 13\% of patients undergoing transplantation or receiving an LVAD $(\mathrm{n}=232)$ during the same time period. The technique was used in 14 transplant recipients $(48 \%)$ and 15 LVAD recipients $(52 \%)$. Among the LVADs, the average Interagency Registry for Mechanically Assisted Circulatory Support category was 2 , with $7(46.7 \%)$ requiring intraaortic balloon pump and $14(93.3 \%)$ requiring inotropic support preoperatively. Of the 14 transplant recipients, 9 $(64 \%)$ received skin only closure and $5(36 \%)$ received a bridging plate. Of the 15 LVAD recipients, $9(60 \%)$ received skin only closure and $6(40 \%)$ received a bridging. All patients underwent chest closure in the operating room once stabilized. No patient experienced a sternal wound, mediastinal, or LVAD pocket infection during the index hospitalization. Informed consent from the patient was not obtained because no patient specific data is reported.

\section{COMMENT}

This technique is useful when leaving the chest open because it allows for sternal stability and maintenance of a biological barrier. As the number/complexity of LVAD and transplants increases, surgeons will encounter this problem more frequently, especially programs that have expanded selection criteria to include high-risk donor hearts or LVAD recipients with compromised right ventricular function. These populations are at higher risk for right ventricular dysfunction and the associated coagulopathy commonly seen with hepatic congestion. Although not all patients require this approach, many surgeons keep the chest open in such situations to allow for coagulopathy correction and hemodynamic stabilization.
TABLE 1. Demographic characteristic of patients with delayed sternal closure using described surgical technique

\begin{tabular}{lc}
\hline \multicolumn{1}{c}{ Characteristic } & Value (n=29) \\
\hline Mean age, y (range) & $56(21-76)$ \\
Gender & \\
$\quad$ Female & $2(7 \%)$ \\
$\quad$ Male & $27(93 \%)$ \\
Primary procedure & \\
$\quad$ Cardiac transplant (\%) & $14(48 \%)$ \\
$\quad$ LVAD (\%) & $15(52 \%)$ \\
Diagnosis & \\
$\quad$ Ischemic cardiomyopathy (\%) & $16(55 \%)$ \\
$\quad$ Nonischemic cardiomyopathy $(\%)$ & $13(45 \%)$ \\
Redo sternotomy $(\%)$ & $6(21 \%)$ \\
Mean cardiopulmonary bypass time (min) & 186 \\
Bridging plate used (\%) & $11(38 \%)$ \\
\hline
\end{tabular}

LVAD, Left ventricular assist device.

Few studies have investigated the impact of delayed sternal closure on outcomes, but no study has evaluated the impact of technique. Most studies have used techniques involving synthetic materials to bridge the sternum. ${ }^{1}$ Other studies have shown good results with sewing a substernal pericardial patch combined with soft tissue closure. ${ }^{2}$ Our approach, involving a bridging plate along with skin reapproximation, is unique, and we believe further evaluation in the form of a multicenter study might be of value. Although little is known about infection risk with different techniques, a biologic barrier affords the lowest risk theoretically because it allows the patients immune system to protect the wound. A study of lung transplants managed with delayed chest closure identified skin reapproximation as the superior approach with regard to mortality outcomes compared with synthetic material. ${ }^{3}$ These data further support the benefits of a biologic barrier, especially in immunocompromised patients.

One unique aspect of our approach is avoiding packing material between the heart and the sternum. We have found its exclusion to be both safe and effective in our limited series. We routinely place a large piece of Gelfoam between the heart and the sternum in those patients whose right ventricle approaches or contacts the sternum, effectively creating a barrier and preventing contact abrasion without compromising hemodynamics. We have had no incidence of cardiac injury using this approach.

\section{CONCLUSIONS}

We have used this technique for 29LVAD or transplant recipients and found it to be a simple and efficient way to perform delayed sternal closure while minimizing infectious risk.

\section{References}

1. Fanning WJ, Vasko JS, Khan JW. Delayed sternal closure after cardiac surgery. Ann Thorac Surg. 1987;44:169-72. 
2. Rajakaruna C, Rodriguez GM, Rajbanshi BG, Ziganshin BA, Elefteriades JA. Novel technique for delayed sternal closure: soft tissue approximation with substernal bridge. J Thorac Cardiovasc Surg. 2014;148:2427-9.
3. Shigemura N, Orhan Y, Bhama JK, D’Cunha J, Zaldonis D, Pilewski JM, et al. Delayed chest closure after lung transplantation: techniques, outcomes, and strategies. J Heart Lung Transplant. 2014;33:741-8. 